\title{
Clinical pathology of metastatic gastric carcinoma to the breast: A report of two cases and a review of literature
}

\author{
QIUHONG TIAN $^{1}$, JINSHENG ZENG ${ }^{2}$, XUEQIN TAO $^{3}$, ZHANMIN ZHANG $^{1}$, \\ $\mathrm{XIAODONG} \mathrm{ZHOU}^{4}$ and YITING WANG ${ }^{1}$ \\ Departments of ${ }^{1}$ Oncology, ${ }^{2}$ Surgery, ${ }^{3}$ Pathology and ${ }^{4}$ Gastroenterology, \\ First Affiliated Hospital of Nanchang University, Nanchang, Jiangxi 330006, P.R. China
}

Received March 13, 2015; Accepted December 11, 2015

DOI: $10.3892 / 01.2016 .4350$

\begin{abstract}
The breast is an unusual site for metastasis from a gastric carcinoma. The present study reports two cases of metastatic gastric carcinoma to the breast. The first patient, a 37-year-old woman, initially presented with gastric adenocarcinoma, prior to developing metastatic cancer to the breast 4 years later. The second female patient presented with a breast mass, and a modified radical mastectomy was performed; however, the subsequent pathological examination revealed the mass to be a metastatic signet ring cell carcinoma. An abdominal computed tomography scan revealed a diffuse gastric wall thickening that was consistent with gastric cancer. The findings suggest that immunohistochemistry is a useful tool to differentiate between primary breast tumors and gastrointestinal carcinomas that have metastasized to the breast. Additional studies are required in order to define the optimal treatment.
\end{abstract}

\section{Introduction}

Breast cancer is the most common type of cancer among females, with an estimated annual incidence of 1.6 million cases, worldwide (1). The majority of breast cancer patients are diagnosed during the early stages, during which treatment is generally effective, with a 5-year survival rate of almost $90 \%$. However, breast cancer also has a high recurrence rate, with up to $40 \%$ of patients eventually progressing to metastatic breast cancer. Compared to early stage breast cancer, metastatic breast cancer is associated with a much more negative prognosis, with a 5-year survival rate of only $25 \%$ (2).

Secondary breast carcinomas are extremely rare. The first case of metastasis to the breast was reported in an autopsy study

Correspondence to: Dr Jinsheng Zeng, Department of Surgery, First Affiliated Hospital of Nanchang University, 17 Yongwai Zheng Street, Nanchang, Jiangxi 330006, P.R. China

E-mail: jinshengzeng23@yeah.net

Key words: gastric carcinoma, immunohistochemistry, breast metastasis in 1957 (3). Alva and Shetty-Alva (4) reported that metastases to the breast have been identified at rates of 1.7-6.6\% based on autopsy studies of patients with any type of cancer, $1.2-2 \%$ in clinical case reports and $2.7 \%$ in cytological reviews.

Commonly, metastatic breast cancer is an incurable disease, and treatment aims to prolong survival and to improve or maintain quality of life by palliating disease-associated symptoms while minimizing the toxicity of treatment. The median survival time of metastatic breast cancer patients is 2 years (5). Patients with hormone-insensitive disease and patients that do not respond to endocrine therapy are candidates for chemotherapy (6). Combination chemotherapy provides higher response rates and increased time to progression. Newer taxane-containing combination regimens, including docetaxel/capecitabine and paclitaxel/gemcitabine, have been demonstrated to improve overall survival compared with single-agent taxanes, and these regimens are commonly used when a combination therapy is adopted. However, the optimal duration of treatment to control disease with these regimens remains to be elucidated. In a previous meta-analysis, Gennari et al (6) reported that increased first-line chemotherapy duration was associated with prolonged progression-free survival and marginally longer overall survival. Continuing chemotherapy until disease progression ceases is additionally reported to improve quality of life measures. Capecitabine is an oral fluoropyrimidine and has been demonstrated to have marked activity in metastatic breast cancer (7).

Longer survival of patients with metastatic breast cancer and the use of improved imaging techniques are associated with an increased incidence of brain metastases. Patients who develop brain metastases tend to have poor prognosis, with short overall survival times. In addition, brain metastases are a significant cause of morbidity, associated with progressive neurological deficits that result in a reduced quality of life. Current therapies include surgery, whole-brain radiation therapy, stereotactic radiosurgery, chemotherapy and targeted therapies. However, the timing and appropriate use of these therapies is controversial and careful patient selection by using available prognostic tools is extremely important (8).

In the current study, a retrospective analysis that assessed cases of metastases to the breast from a gastric carcinoma, diagnosed between January 2007 and April 2013, was performed, and two patients were identified. The clinical and pathological 
features of the aforementioned metastasis are presented herein, in order to gain insight into the clinical presentation and natural history of this disease. Consent for the publication of patient data was obtained from the next of kin.

\section{Case report}

Case one. In January 2007, a 37-year-old woman presented to the First Affiliated Hospital of Nanchang University (Nanchang, Jiangxi, China) with upper abdominal pain that had lasted for 2 months. Computed tomography indicated a diagnosis of signet ring cell carcinoma, and the patient underwent a radical gastrectomy. The subsequent pathological examination revealed a level II gastric adenocarcinoma with an ulcerative signet ring cell histology. Metastasis was identified in 2/8 lymph nodes along the lesser curvature of the stomach. Subsequent to surgery, the patient received two 3-week cycles of chemotherapy with paclitaxel (210 mg, day 1 of each cycle) and cisplatin (30 mg, days 1-3 of each cycle).

In October 2009, a radical colectomy was performed on the right half of the colon due to the presence of colonic metastasis, and was followed by 1 cycle ( 3 weeks) of chemotherapy with oxaliplatin (200 mg, day 1 of each cycle), calcium folinate $(0.6 \mathrm{~g} /$ day, day 1 of the cycle) and 5-fluorouracil (3.75 g/day, $46 \mathrm{~h}$ intravenous administration, day 1 of the cycle). In June 2011, four years since the gastrectomy was performed, the patient presented to the same hospital with a 9x5 cm left upper outer quadrant and a $3 \times 2 \mathrm{~cm}$ left lower outer quadrant breast mass. The masses were movable, smooth with irregular margins and exhibited slight tenderness. Two asymptomatic left axillary lymph nodes were palpable. A mammography examination revealed dense left breast glands with malignant changes and hyperplasia, and the breast ultrasound demonstrated hypoechoic lesions in the left breast and the two axillas. The right breast appeared normal.

A breast biopsy indicated the presence of a signet ring cell carcinoma, and a modified radical mastectomy was performed. A hard, gray mass, measuring $10 \times 7 \times 4 \mathrm{~cm}$, had replaced the majority of the breast tissue and invaded the nipple. The postoperative pathological diagnosis was signet ring cell carcinoma (Fig. 1A). An intravascular tumor thrombus was present, and metastasis was identified in 17/21 of the axillary lymph nodes. Immunohistochemical staining of the tumor revealed the following: Cytokeratin (CK) 7(2+) (Fig. 1B), CK20(-), villin(2+) (Fig. 1C), carcinoembryonic antigen (CEA)(3+) (Fig. 1D), caudal type homeobox 2(-), estrogen receptor (ER)(-), progesterone receptor $(\mathrm{PR})(-)$ and receptor tyrosine-protein kinase Erb-B2 (HER2)(-). The patient refused postoperative treatment and succumbed to the disease 18 months later, in December 2012.

Case two. In February 2013, a 31-year-old woman presented to the First Affiliated Hospital of Nanchang University with a $6.5 \times 6.4 \mathrm{~cm}$ mass in the right breast, and an ultrasound confirmed a hypoechoic mass. The mammography revealed retraction of the right nipple and dense glands. A biopsy of the breast revealed an invasive carcinoma.

A modified radical mastectomy of the right breast was performed. A subsequent pathological examination revealed a metastatic signet ring cell carcinoma (Fig. 2A), and metastasis was identified in $7 / 15$ of the axillary lymph nodes.
Immunohistochemical evaluation of the tumor yielded the following staining results: CK7(-), CK20(3+) (Fig. 2B), villin(3+) (Fig. 2C), S100(-), Vim(-), ER(-), PR(-), c-Erb-B2(-), Ki-67 (80\%, $+)$, P53(-), CD34(-) and E-cadherin(2+). An abdominal computed tomography scan was performed, the results of which revealed a diffuse gastric wall thickening, consistent with gastric cancer. A gastroscopy revealed the presence of linitis plastica (Fig. 2D) and a biopsy indicated the presence of signet ring cell carcinoma. The patient was diagnosed with stage IV gastric cancer, and 2 cycles of chemotherapy with paclitaxel (270 mg, day 1) and cisplatin (40 mg, days 1-3) were administered. The patient succumbed to the disease in November 2013.

\section{Discussion}

The most common metastatic tumor to the breast is malignant melanoma, followed by lymphoma, neuroendocrine tumor, thyroid, lung, cervical, ovarian, renal and liver cancers, sarcoma and nasopharyngeal carcinoma (9). Gastric carcinomas metastasizing to the breast are less common; to the best of our knowledge, only 25 cases had been reported in the English literature prior to 2006 (10). Gastric cancer tends to metastasize to hormone-dependent organs, which is likely to be due to the rich blood supply available to these organs. Premenopausal women are most commonly affected by the disease, and a previously reported average age at presentation is 47 years (11). The patients in the current study were younger compared with this figure, with an average age of 34 years.

Gastric carcinoma metastases to the breast are typically signet ring carcinomas, and must be differentiated from primary breast signet ring carcinomas, which were first described as a subtype of lobular tumors by Steinbreche and Silverberg in 1976 (12). Primary breast signet ring cell carcinomas account for $2.0-4.5 \%$ of all breast tumors, are more aggressive compared with other breast tumors and frequently metastasize to the abdomen and gastrointestinal tract (12). Approximately two thirds of secondary tumors present as a single painless mass in the upper outer quadrant of the breast. Nipple discharge is rarer than that observed in primary breast cancer. The disease is similar to inflammatory breast cancer, and edema of the skin is evident.

In previous studies, $25 \%$ of patients with breast metastases possessed bilateral breast tumors and $5 \%$ were found to have axillary lymph node metastases $(10,11)$. By contrast, the patients in the present study had solitary left and right breast masses, respectively, with no pain, and axillary lymph node metastases were confirmed. Toombs and Kalisher (13) reported that $66 \%$ of solitary metastases to the breast were located in the upper outer quadrant. In a retrospective study of 2,414 cases of primary breast cancer, approximately two thirds of the tumors were located in the outer quadrants, and one third were located in the inner quadrants. The position of the breast mass was not found to be useful for the differentiation between primary and secondary breast cancer (14).

Immunohistochemistry is useful for the differentiation of primary or metastatic breast signet ring cell carcinomas. Primary breast signet ring cell carcinomas occur most frequently in premenopausal women and have high expression levels of ER, PR and gross cystic disease fluid protein (15). The pathological features of metastatic carcinomas to the 
A

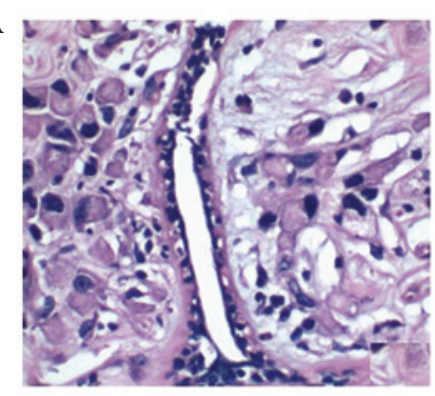

C

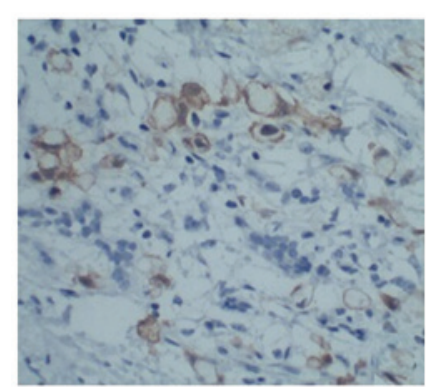

B

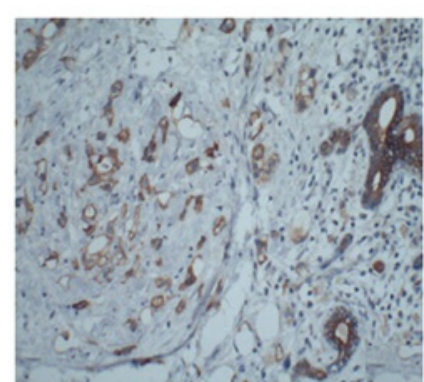

D

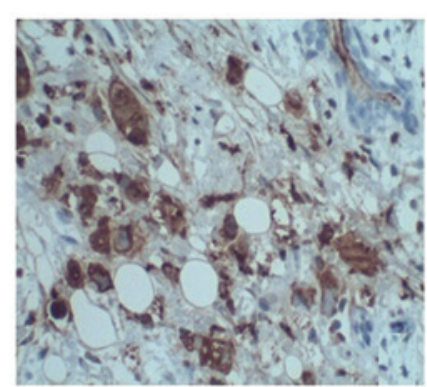

Figure 1. Case one. Left breast with metastatic signet ring cell carcinoma: (A) Hematoxylin and eosin stain and immunohistochemical stains revealing the expression of (B) cytokeratin 7, (C) villin and (D) carcinoembryonic antigen (magnification, $\mathrm{x} 400$ ).

\section{A}

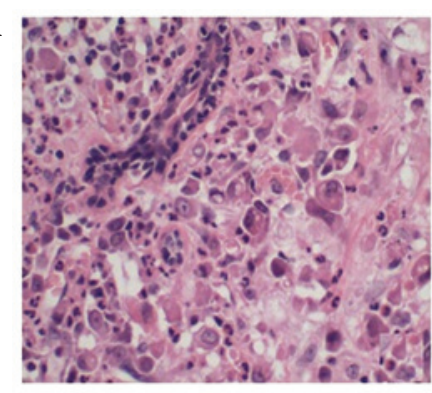

B

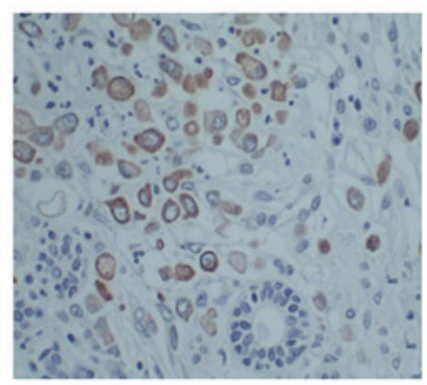

C

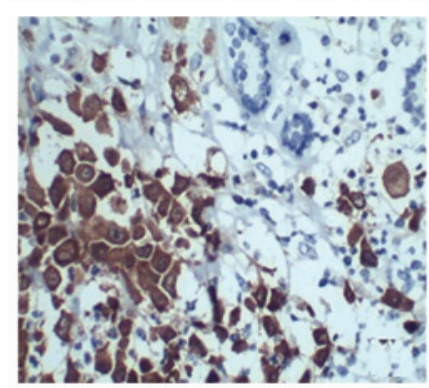

D

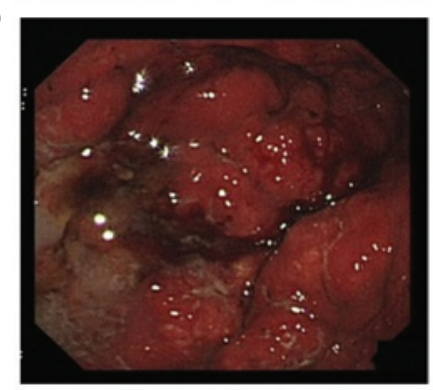

Figure 2. Case two. Right breast with metastatic signet ring cell carcinoma: (A) Hematoxylin and eosin stain and immunohistochemical stains revealing the expression of (B) cytokeratin 20 and (C) villin (magnification, x400). (D) Linitis plastica revealed by gastroscopy.

breast include the replacement of normal breast tissue by malignancy and the invasion of the adjacent normal tissue. Lobular carcinomas in situ and intraductal tumors are not observed in metastatic disease (10). Metastatic tumors are generally similar to their primary tumors based on immunohistochemistry, which may be useful in discerning whether breast tumors are primary or metastatic. Villin, for example, is a gastrointestinal cytoskeletal protein present in brush border microvilli that is not present in breast tissue. In addition, metastases from adenocarcinoma of the stomach express the skin markers CEA, CK7 and CK20, while ER and PR are not expressed (15). HER2 is expressed in $20 \%$ of gastric cancers and is not specific to breast cancer (16).
Microcalcifications within breast metastases are not commonly observed on axial imaging. Only a few cases of microcalcifications associated with breast metastases from gastric cancer have been reported in the literature (17). In 2012, Luk et al (18) described the case of a patient who presented with a breast mass that contained microcalcifications, which was later confirmed to be metastasized from a gastric carcinoma.

A breast lumpectomy is currently the recommended treatment. Furthermore, previous evidence has indicated that radiotherapy may not be effective in the treatment of breast metastases (11). The systemic treatment of the primary cancer is necessary, and a resection of the primary tumor may prolong 
the survival of patients with gastric carcinoma metastatic to the breast.

Breast metastases from gastric carcinomas are associated with a poor prognosis, and the majority of patients succumb within 1 year (19). The survival of patients with breast metastases from gastric carcinomas is significantly decreased compared with patients with primary breast cancer (20). Breast metastases from gastric carcinoma are rare; thus, increasing awareness of the disease is important in order to avoid misdiagnosis. Additional studies are required to define the best treatment.

\section{Acknowledgements}

The present study was supported by the foundation grant from JiangXi Province Scientific and Technological Bureau (no. 20112BBG70051).

\section{References}

1. Cuzick J, Sestak I, Bonanni B, Constantino JP, Cummings S, DeCensi A, Dowsett M, Forbes JF, Ford L, LaCroix AZ, et al SERM Chemoprevention of Breast Cancer Overview Group: Selective oestrogen receptor modulators in prevention of breast cancer: An updated meta-analysis of individual participant data. Lancet 381: 1827-1834, 2013.

2. National Cancer Institute. Surveillance, Epidemiology and End Results Stat Fact Sheets: Female Breast Cancer. National Cancer Institute, Bethesda, MD, USA, pp15-16, 2014.

3. Kashlan RB, Powell RW and Nolting SF: Carcinoid and other tumors metastatic to the breast. J Surg Oncol 20: 25-30, 1982.

4. Alva S and Shetty-Alva N: An update of tumor metastasis to the breast data. Arch Surg 134: 450, 1999.

5. Albain KS, Nag SM, Calderillo-Ruiz G, Jordaan JP, Llombart AC, Pluzanska A, Rolski J, Melemed AS, Reyes-Vidal JM, Sekhon JS, et al: Gemcitabine plus paclitaxel versus paclitaxel monotherapy in patients with metastatic breast cancer and prior anthracycline treatment. J Clin Oncol 26: 3950-3957, 2008.

6. Gennari A, Stockler M, Puntoni M, Sormani M, Nanni O, Amadori D, Wilcken N, D'Amico M, DeCensi A and Bruzzi P: Duration of chemotherapy for metastatic breast cancer: A systematic review and meta-analysis of randomized clinical trials. J Clin Oncol 29: 2144-2149, 2011.

7. O'Shaughnessy JA, Kaufmann M, Siedentopf F, Dalivoust P, Debled M, Robert NJ and Harbeck N: Capecitabine monotherapy: Review of studies in first-line HER-2-negative metastatic breast cancer. Oncologist 17: 476-484, 2012.
8. Arslan C, Dizdar O and Altundag K: Systemic treatment in breast-cancer patients with brain metastasis. Expert Opin Pharmacother 11: 1089-1100, 2010.

9. Domanski HA: Metastases to the breast from extramammary neoplasms. A report of six cases with diagnosis by fine needle aspiration cytology. Acta Cytol 40: 1293-1300, 1996.

10. Boutis AL, Andreadis C, Patakiouta F and Mouratidou D: Gastric signet-ring adenocarcinoma presenting with breast metastasis. World J Gastroenterol 12: 2958-2961, 2006.

11. Qureshi SS, Shrikhande SV, Tanuja S and Shukla PJ: Breast metastases of gastric signet ring cell carcinoma: A differential diagnosis with primary breast signet ring cell carcinoma. J Postgrad Med 51: 125-127, 2005.

12. Steinbrecher JS and Silverberg SG: Signet-ring cell carcinoma of the breast. The mucinous variant of infiltrating lobular carcinoma? Cancer 37: 828-840, 1976.

13. Toombs BD and Kalisher L: Metastatic disease to the breast: Clinical, pathologic, and radiographic features. AJR Am J Roentgenol 129: 673-676, 1977.

14. Janni W, Rack B, Sommer H, Schmidt M, Strobl B, Rjosk D, Klanner E, Thieleke W, Gerber B, Friese K and Dimpfl T: Intra-mammary tumor location does not influence prognosis but influences the prevalence of axillary lymph-node metastases. J Cancer Res Clin Oncol 129: 503-510, 2003.

15. Briest S, Horn LC, Haupt R, Schneider JP, Schneider U and Höckel M: Metastasizing signet ring cell carcinoma of the stomach-mimicking bilateral inflammatory breast cancer. Gynecol Oncol 74: 491-494, 1999.

16. Gravalos C and Jimeno A: HER2 in gastric cancer: A new prognostic factor and a novel therapeutic target. Ann Oncol 19: 1523-1529, 2008

17. Lee SK, Kim WW, Kim SH, Hur SM, Kim S, Choi JH, Cho EY, Han SY, Hahn BK, Choe JH, et al: Characteristics of metastasis in the breast from extramammary malignancies. J Surg Oncol 101: 137-140, 2010.

18. Luk YS, Ka SY, Lo SS, Chu CY and Ma MW: An unusual case of gastric cancer presenting with breast metastasis with pleomorphic microcalcifications. J Breast Cancer 15: 356-358, 2012.

19. Parrell Soler C, Palacios Marqués A, Saco López L, Bermejo De Las Heras R and Pertusa Martínez S: Breast metastatic localization of signet-ring cell gastric carcinoma. ISRN Obstet Gynecol 2011: 426150, 2011.

20. Zibari GB, Riche A, Zizzi HC, McMillan RW, Aultman DF, Boykin KN, Gonzalez E, Nandy I, Dies DF, Gholson CF, et al: Surgical and nonsurgical management of primary and metastatic liver tumors. Am Surg 64: 211-220; discussion, 220-221, 1998. 